ADVANCED ELECTRIC AND MAGNETIC MATERIAL MODELS FOR FDTD ELECTROMAGNETIC CODES

B. R. Poole, S. D. Nelson, S. Langdon

May 9, 2005

2005 Particle Accelerator Conference (PAC 2005) Knoxville, TN, United States

May 16, 2005 through May 29, 2005 
This document was prepared as an account of work sponsored by an agency of the United States Government. Neither the United States Government nor the University of California nor any of their employees, makes any warranty, express or implied, or assumes any legal liability or responsibility for the accuracy, completeness, or usefulness of any information, apparatus, product, or process disclosed, or represents that its use would not infringe privately owned rights. Reference herein to any specific commercial product, process, or service by trade name, trademark, manufacturer, or otherwise, does not necessarily constitute or imply its endorsement, recommendation, or favoring by the United States Government or the University of California. The views and opinions of authors expressed herein do not necessarily state or reflect those of the United States Government or the University of California, and shall not be used for advertising or product endorsement purposes. 


\title{
ADVANCED ELECTRIC AND MAGNETIC MATERIAL MODELS FOR FDTD ELECTROMAGNETIC CODES *
}

\author{
Brian R. Poole, Scott D. Nelson, LLNL, Livermore, CA 94550, U.S.A. \\ Scott Langdon, REMCOM Inc, State College, PA 16801, U.S.A.
}

\begin{abstract}
The modeling of dielectric and magnetic materials in the time domain is required for pulse power applications, pulsed induction accelerators, and advanced transmission lines. For example, most induction accelerator modules require the use of magnetic materials to provide adequate Volt-sec during the acceleration pulse. These models require hysteresis and saturation to simulate the saturation wavefront in a multipulse environment. In high voltage transmission line applications such as shock or soliton lines the dielectric is operating in a highly nonlinear regime, which require nonlinear models. Simple 1-D models are developed for fast parameterization of transmission line structures. In the case of nonlinear dielectrics, a simple analytic model describing the permittivity in terms of electric field is used in a 3-D finite difference time domain code (FDTD). In the case of magnetic materials, both rate independent and rate dependent Hodgdon magnetic material models have been implemented into 3-D FDTD codes and 1-D codes.
\end{abstract}

\section{INTRODUCTION}

The interaction of transient high power electromagnetic pulses with materials requires the inclusion of nonlinear effects in the constitutive relation for the material. For example, nonlinear dielectrics or nonlinear ferrites are utilized in electromagnetic shock lines to sharpen the leading edge of a pulse [1]. Nonlinear materials can also be used in periodic arrays in transmission lines to generate solitons where the "balance" of dispersion and nonlinearity is used to propagate pulses with minimal distortion [2]. Understanding the effect of saturation and hysteresis in a magnetic ferrite is of importance in the design of induction accelerator cells in order to insure a minimal leakage current. In this paper we discuss the inclusion of a simple nonlinear permittivity model and the Hodgdon magnetic material model [3] into a FDTD electromagnetic code.

\section{MODELING}

The inclusion of realistic models of the constitutive properties of electric and magnetic materials is a requisite for the proper design of electromagnetic structures. For pulse power applications, when dealing with short high amplitude electromagnetic pulses it is required to incorporate nonlinear models of the materials into modeling codes. The electromagnetic modeling code

*This work performed under the auspices of the U.S. Department of Energy by the University of California, Lawrence Livermore National Laboratory under Contract No. W-7405-Eng-48.
XFDTD [4] has been used extensively for modeling transient linear electromagnetics. It incorporates the unique feature of allowing lumped circuit elements, including resistors, capacitors, inductors, and opening and closing switches to be incorporated into the model making it a useful tool for pulse power applications. The code includes models for, lossy anisotropic dielectrics, and Debye and Lorentzian dielectric dispersion. Magnetic models include magnetized ferrites, and Debye dispersion. For high field applications models for nonlinear anisotropic dielectrics and saturable magnetic materials with hysteresis were developed for XFDTD. Several test problems were set up in XFDTD in a geometry that approximated a 1-D problem. Doing this allowed us to use the 1-D PDE solver, FEMLAB [5] to compare solutions from the two independent solvers to check the validity of the implementation in XFDTD.

\section{Nonlinear Dielectric Materials}

Eq.1 defines the nonlinear permittivity function implemented in XFDTD. It was chosen to provide a sufficient number of free parameters to model a variety of dielectric materials,

$$
\varepsilon=\varepsilon_{2}+\frac{\varepsilon_{1}-\varepsilon_{2}}{1+a_{1}\left(E / E_{0}\right)^{2}+a_{2}\left(E / E_{0}\right)^{4}+a_{3}\left(E / E_{0}\right)^{6}}
$$

where $\varepsilon_{1}, \varepsilon_{2}, E_{0}$, and $a_{i}$ are parameters used to define the nonlinear function.

Figure 1 shows a simple classical problem consisting of a parallel plate transmission line with a block of nonlinear material inserted between the plates.

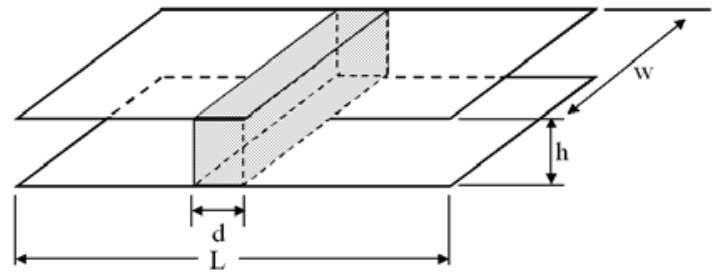

Figure 1: Stripline with block of nonlinear dielectric.

If the plates are sufficiently wide compared with the distance between the plates, the problem is approximately 1-D and can be modeled by 1-D Maxwell equations. The dimensions of the stripline are; $L=400 \mathrm{~cm}, d=50 \mathrm{~cm}$, $w=9 \mathrm{~cm}$, and $h=3 \mathrm{~mm}$. The parameters for the dielectric material are; $\varepsilon_{1}=4, \varepsilon_{2}=1, a_{1}=1, a_{2}=a_{3}=0$, and $E_{0}=$ $100 \mathrm{kV} / \mathrm{m}$. Figure 2 shows a plot of the nonlinear 
dielectric function. The line is driven with a $100 \mathrm{kV} / \mathrm{m}$ Gaussian pulse with a FWHM of 1 ns at the left end of the transmission line.

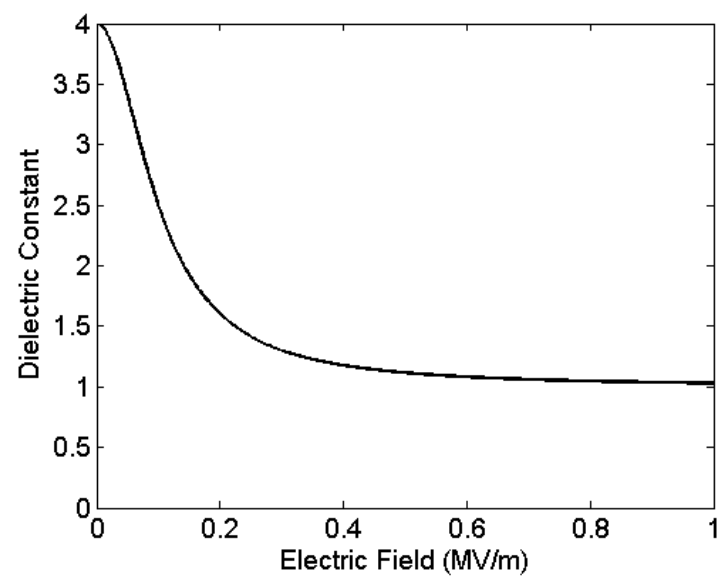

Figure 2: Nonlinear dielectric function for test problem.

Figures 3 and 4 show the temporal evolution of the electric field pulse $25 \mathrm{~cm}$ before and after the material. Figure 3 shows the $100 \mathrm{kV} / \mathrm{m}$ incident pulse along with reflections from the front and back edges of the material. The reflection from the back surface shows the shock front produced by traversal through the material.

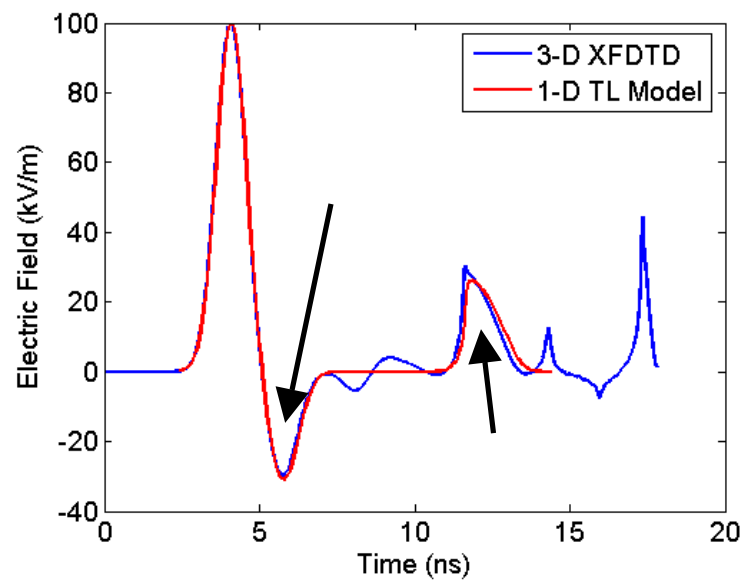

Figure 3: Electric field $25 \mathrm{~cm}$ before dielectric insert.

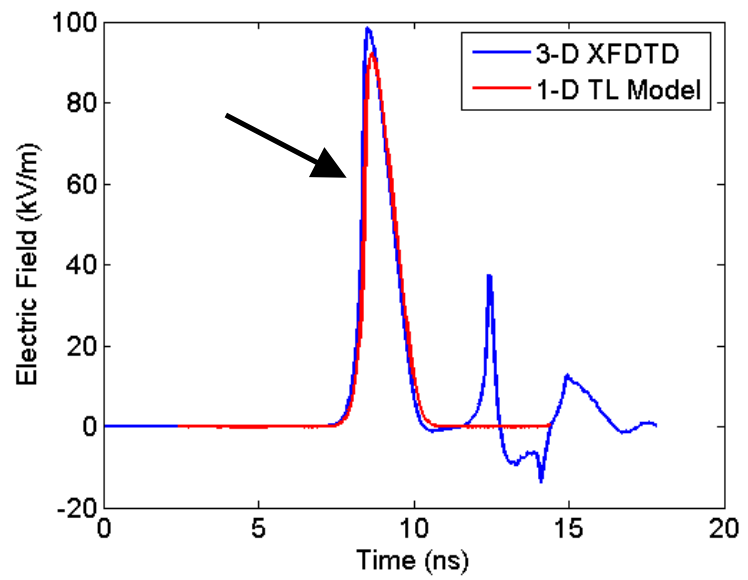

Figure 4: Electric field $25 \mathrm{~cm}$ after dielectric insert.
Figure 4 again clearly shows the development of the sharpened rise time produced by the nonlinear dielectric. The late time behavior in the 3-D results from a poorly matched load at the right side of the transmission line. The 1-D calculations are useful to parameterize or optimize a system before utilizing a full 3-D description of the problem since the 3-D calculations take a substantially longer time.

\section{Saturable Magnetic Materials and Hysteresis}

To model ferromagnetic hysteresis, both the rate independent and rate dependent Hodgdon magnetic material models [3] were implemented into the XFDTD code. The implementation of the Hodgdon material model is substantially more difficult than the nonlinear dielectric model, since in that case the dielectric permittivity is expressed explicitly as a function of the electric field while in the case of the Hodgdon model, the permeability is described by a nonlinear differential equation in terms of the magnetic field and the magnetic flux vector. Eq. 2 defines the permeability in terms of $B, H$, and $\dot{B}$.

$$
\mu(B, H, \dot{B})=\{\alpha \operatorname{sgn}(\dot{B})[f(B)-H]+g(B, \dot{B})\}^{-1}
$$

where

$$
f(B)= \begin{cases}A_{1} \tan A_{2} B, & |B| \leq B_{b p} \\ A_{1} \tan A_{2} B_{b p}+\left(B-B_{b p}\right) / \mu_{s}, & B>B_{b p} \\ -A_{1} \tan A_{2} B_{b p}+\left(B+B_{b p}\right) / \mu_{s}, & B<-B_{b p}\end{cases}
$$

and

$$
g(B, \dot{B})=f^{\prime}(B)\left[1-A_{3} c(\dot{B}) \exp \left(-\frac{A_{4}|B|}{B_{c l}-|B|}\right)\right]
$$

if $|B| \leq B_{c l}$ and $g(B, \dot{B})=f^{\prime}(B)$ otherwise. The term, $c(\dot{B})$ is defined by

$$
c(\dot{B})= \begin{cases}1+c_{1}|\dot{B}|, & |\dot{B}|<\dot{B}_{1} \\ 1+c_{1} \dot{B}_{1}+c_{2}\left(|\dot{B}|-\dot{B}_{1}\right), & \dot{B}_{1} \leq|\dot{B}| \leq \dot{B}_{2} \\ 1+c_{1} \dot{B}_{1}+c_{2}\left(\dot{B}_{2}-\dot{B}_{1}\right) & \\ +c_{3}\left(|\dot{B}|-\dot{B}_{2}\right), & |\dot{B}|>\dot{B}_{2}\end{cases}
$$

Here the constants $\alpha, \mu_{s}, A_{i}, B_{b p}, B_{c l}$, and the function $c(\dot{B})$ are material parameters as described in 
[3]. The function $c(\dot{B})$ defines whether the model is rate independent $\left(c_{i}=0\right)$ or rate dependent $\left(c_{i} \neq 0\right)$.

Figure 5 shows a simple coaxial geometry with a $2 \mathrm{~cm}$ thick ferrite disk inserted into the transmission line. The code simulations were done for CN20 ferrite. The inner and outer radius of the line is $8 \mathrm{~cm}$ and $10 \mathrm{~cm}$ respectively.

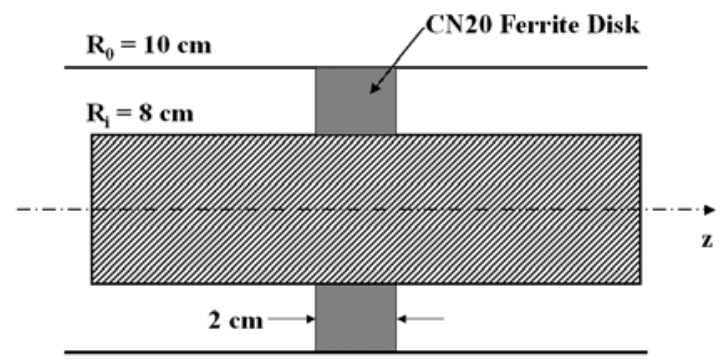

Figure 5: Coaxial transmission line with ferrite insert.

A reasonable 1-D approximation for this problem can be made since the magnetic flux lines close within the material and the ratio of outer to inner conductor radius is only slightly larger than unity. This last approximation is required to insure that the fields are relatively uniform across the radius and hence remain primarily TEM in the structure. The rate independent parameters for CN20 ferrite are; $B_{c l}=0.4 \mathrm{~T}, B_{b p}=.4103 \mathrm{~T}, H_{c l}=397.9, \mu_{s}=$ $2 \mu_{0}, \alpha=10 \mathrm{~T}^{-1}, A_{1}=21.19 \mathrm{Amp} / \mathrm{m}, A_{2}=3.794 \mathrm{~T}^{-1}$, $A_{3}=-4.489$, and $A_{4}=.3046$. The rate dependent parameters are; $c_{1}=13.3 \times 10^{-7}(\mathrm{~T} / \mathrm{s})^{-1}, c_{2}=8 \times 10^{-7}(\mathrm{~T} / \mathrm{s})^{-1}$, $\dot{B}_{1}=7.5 \times 10^{5}(\mathrm{~T} / \mathrm{s})$ and $\dot{B}_{2} \rightarrow \infty$. A TEM Gaussian pulse of approximate amplitude $314 \mathrm{kV} / \mathrm{m}$ and FWHM of 6.65 ns is incident on the ferrite from the left. Figure 6 shows a plot of the hysteresis loop determined by direct integration of Eq. 2.

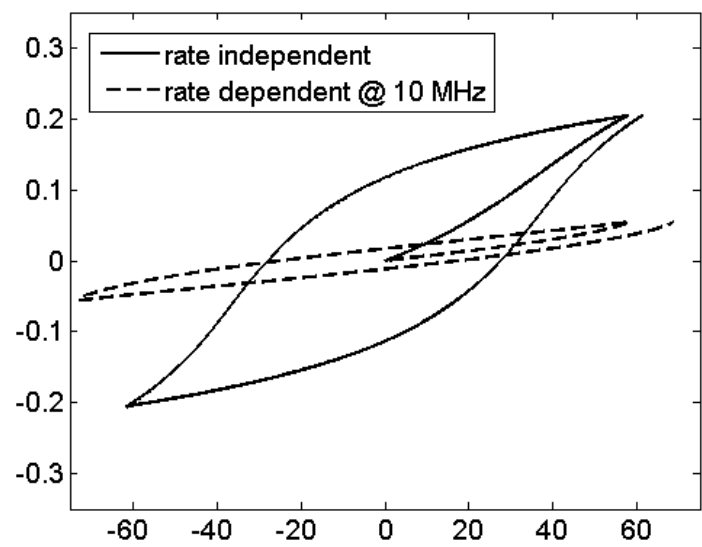

Figure 6: Hysteresis curves for CN20 ferrite

In the linear regime, the material exhibits a permeability of approximately 1800 for the rate independent calculation and 497 atr $10 \mathrm{MHz}$. Similar calculations show a permeability of 132 at $200 \mathrm{MHz}$. The rate dependent results clearly show a dramatic effect in the reduction of the permeability. Figure 7 shows the transmitted pulse through the geometry shown in Fig. 5 for the 1-D and 3-D rate independent calculations. Figure 8 shows a similar calculation for a rate dependent problem.

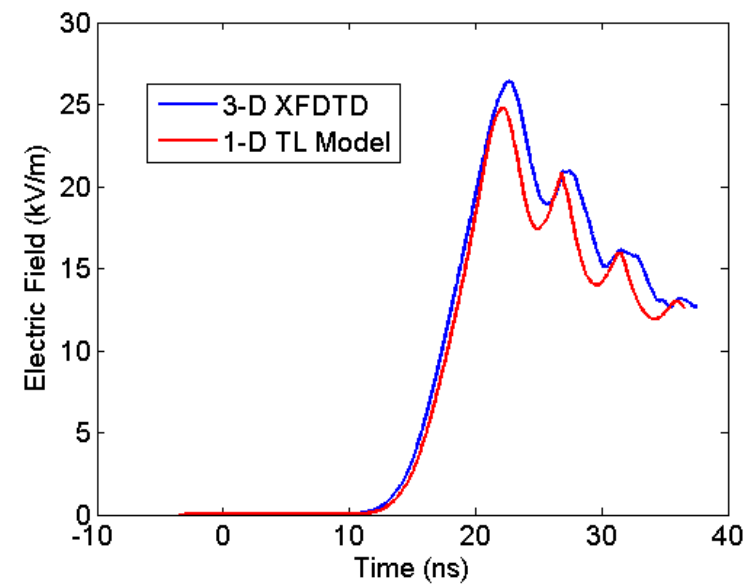

Figure 7: Electric field $1.1 \mathrm{~cm}$ after ferrite insert.

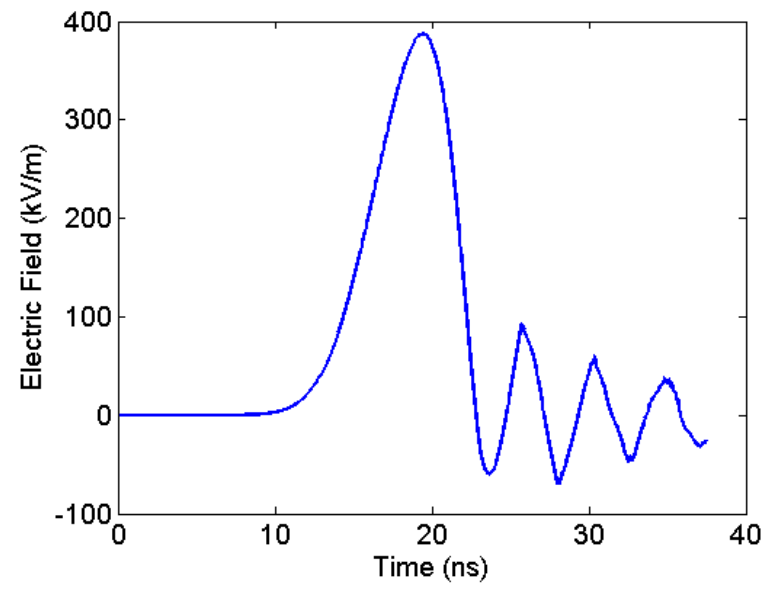

Figure 8: Electric field $1.1 \mathrm{~cm}$ after ferrite insert.

Fig 8 shows the effect of rate dependence on the results.

\section{REFERENCES}

[1] G. Branch and P. W. Smith, "Fast-Rise-Time Electromagnetic Shock Waves in Nonlinear, Ceramic Dielectrics," J. Phys. D: Appl. Phys, 29, August 1996, p. 2170-2178.

[2] M. N. Rosenbluth and R. E. Waltz, "Strong Lumped Circuit Solitons,” J. Appl. Phys, 65, May 1989, p3698-3705.

[3] C. D. Boley and M. L. Hodgdon, "Model and Simulation of Hysteresis in Magnetic Cores," Ieee Trans. on Magnetics, September 1989, p. 3922-3924.

[4] XFDTD code developed by REMCOM Corp. http://www.remcom.com.

[5] FEMLAB code developed by COMSOL Corp. http://www.comsol.com. 